\title{
Experiencias en un modelo con material instruccional en línea, casas universitarias y centrado en el aprendizaje: el caso del Centro Universitario de los Valles de la Universidad de Guadalajara
}

\section{Experiences in a model centered on learning with instructional materials online and university residence: the case of the University Center of the Valleys of the University of Guadalajara}

\author{
Mario Martínez García ${ }^{1}$ \\ ${ }^{1}$ Departamento de Ciencias Computacionales e Ingenierías, Universidad de Guadalajara, México \\ (mariom@valles.udg.mx)
}

Recibido el 22 de abril de 2015; revisado el 5 de junio de 2015; aceptado el 2 de junio de 2015; publicado el 5 de junio de 2015

\section{RESUMEN:}

Por su misma naturaleza, el éxito en la formación universitaria depende de varios actores y factores, cuyo desempeño facilita o inhibe el aprendizaje. La relación pedagógica en los cursos presenciales optimizados que se apoyan en las tecnologíasmodelo desarrollado en el Centro Universitario de los Valles (en adelante CU Valles) es afectada por el medio y el contexto de la institución. La calidad de la interacción no solo de los actores centrales del proceso de aprendizaje, estudiantes y profesores, sino también de la misma tecnología, el dispositivo pedagógico utilizado, el apoyo recibido (técnico, académico y social) así como el tiempo dedicado, el lugar y el equipo de trabajo, es decisiva para el buen desarrollo de un curso en línea en este trabajo.

La propuesta contempla la implementación de espacios de gestión de la información y del aprendizaje en línea (Material Instruccional en Línea, en adelante MIEL) y la instalación de espacios equipados con equipo de cómputo y con acceso a Internet en las localidades de la región denominados Comunidades de Aprendizaje y Servicios Académicos (en adelante CASAS Universitarias).
PALABRAS CLAVE: SOCIEDAD DEL CONOCIMIENTO, AMBIENTES VIRTUALES, APRENDIZAJE MODELO, TECNOLOGÍAS DE LA INFORMACIÓN, COMUNICACIÓN, PROGRAMAS EDUCATIVOS.
ABSTRACT:
By its nature, success in university education depends on several actors and factors whose performance facilitates or inhabits learning. The pedagogical relationship optimized classroom courses that rely on technologies-model developed at the Centro Universitario de los Valles (here in after CUValles) - is affected by the medium and context of the institution. The quality of interaction not only of the central actors of the learning process, students and teachers, but also of the same technology, the pedagogic device used, the support (technical, academic and social) as well as time spent, the place and the team is critical to the successful development of an online course in this work.
The proposal includes the implementation of space management information and learning on line (Instructional Material Online, hereinafter MIEL) 
and installation of computer equipment in spaces and access Internet connection in the towns of the region called Learning Communities and Academic Services (here in after CASAS).

$\begin{array}{lrr}\text { KEYWORDS: } & \text { SOCIETY, } & \text { VIRTUAL } \\ \text { ENVIRONMENTS, } & \text { LEARNING } \text { MODEL, } \\ \text { INFORMATION } & \text { TECHNOLOGY, } \\ \text { COMMUNICATION, } & \text { EDUCATIONAL } \\ \text { PROGRAMS. } & \end{array}$

\section{INTRODUCCIÓN}

La transformación del estudiante en la perspectiva de un aprendizaje más eficaz requiere de cambios en su estructura cognitiva y tener acceso a los recursos tecnológicos que le faciliten no solo el acceso a la información sino a medios de comunicación y de trabajo colaborativo y autogestivo como lo menciona (Giordan, 1998), lo cual implica un conocimiento suficiente de las habilidades, estrategias y técnicas que pueden utilizarse, tanto las genéricas como aquellas asociadas directamente a los campos de conocimiento y a las asignaturas (Prieto, 1993). Aprender más eficazmente y aprender a aprender, según (Coll, 1992) son propósitos fundamentales de los sistemas de formación en educación superior, esto se complementa con otros autores como (Hernández, 1991 ) que en la búsqueda de contribuir a la construcción de tejidos sociales además de promover la cultura, las instituciones deben preocuparse por la parte humanista, fomentar el aprender a ser, en la búsqueda de una mayor calidad educativa; para ello, los estudiantes requieren según (Ausubel, 1978):

- Adquirir y utilizar estrategias cognitivas.

- Adquirir y utilizar estrategias metacognitivas.

Utilizar estrategias supone algo más que el conocimiento y la utilización de técnicas o hábitos de estudio, tendencia que se observa en un gran número de instituciones de educación superior. Las estrategias se pueden enseñar y se pueden aprender, (Fly- Jones y Sederburge-Ogle, 1998). La educación, la intervención, el entrenamiento cognitivo, los diversos modelos de enseñanzaaprendizaje favorecen en mayor o menor medida la adquisición y uso de las estrategias cognitivas, (Monereo, 1993). El papel del profesor es fundamental, ya que al explicitar sus objetivos y decidir acerca de las actividades, pero, especialmente, al proporcionar a los estudiantes ciertos mecanismos de ayuda pedagógica, puede favorecer o limitar el aprendizaje de dichas estrategias, (Román-Pérez y Díez-López, 2000).

Aunado a este punto las instituciones de educación están incorporando herramientas informáticas y de redes de comunicación para establecer un enlace entre los contenidos académicos, los estudiantes y los profesores, procurando no perder el contacto humano como lo sugiere (Pascual, 2013) en los modelos mixtos.

Siordia (2011) identifica 3 modelos de formación profesional en los cuales implica el uso de las tecnologías de la información y la comunicación. Estas modalidades son:

- Modelo Adjunto.

- Modelo Mixto.

- Modelo en la red.

El Modelo Adjunto toma como apoyo complementario el uso de herramientas informáticas, si bien no es parte del programa académico, se mejora la experiencia de la formación superior haciendo uso de algunos elementos que propicien el trabajo fuera del horario escolar o curricular, el horario presencial de los estudiantes en las aulas de clase.

El Modelo Mixto considera la incorporación de las tecnologías digitales como parte de la capacitación, el programa de estudios incluye actividades tanto de manera presencial en las instalaciones de la institución educativa, así como actividades en línea, para ello es muy importante que los estudiantes cuenten con acceso a equipos de cómputo conectados a la red.

El Modelo en la red se caracteriza por apoyarse en los medios electrónicos para la totalidad de sus tareas escolares, administrativas y de comunicación (Majo y Marques, 2002), en él se llevan a cabo actividades virtuales para la interacción de los estudiantes con sus asesores, los materiales de apoyo, las dinámicas de aprendizaje, los mecanismos de evaluación están soportados por los entornos electrónicos (Blanco-Méndez y Carlos-Blanco, 2002).

Existen otros modelos que hablan de la interacción entre el profesor y los estudiantes que describe Alonso (1997).

Ante estos panoramas se hace evidente la importancia de incorporar el equipo de cómputo y la Internet en las escuelas para utilizarlas en las actividades educativas, pero no solo basta el hecho de incluir por incluir estas herramientas, sino de facilitarlas a los implicados en el esquema de la enseñanza y del aprendizaje: los profesores y los estudiantes, propiciando modificaciones en los hábitos que consecuentemente con el tiempo 
modifican la cultura y propician una incorporación eficiente de los medios tecnológicos.

La sociedad del siglo XXI requiere profesionistas competitivos, con valores $\mathrm{y}$ habilidades que les permita innovar y adaptarse al cambio constante (Pasturino, 1999), por ello, las Instituciones de Educación Superior tienen la encomienda de establecer estrategias que acerquen las tecnologías del aprendizaje y del acceso a la información para todos aquellos que carecen de ellas desde sus hogares y evitar así el rezago educativo por el simple hecho de ser partícipes en alguno de los 3 modelos antes mencionados.

Son muchas las razones que existen para que las universidades participen en el cambio, entre otras se pueden señalar las siguientes según Bates (2001):

- La necesidad de hacer más por menos.

- Las necesidades de aprendizaje cambiantes de la sociedad.

- El impacto de las nuevas tecnologías para el aprendizaje.

A la par del crecimiento de la educación en línea han surgido diferentes operaciones para crear o diseñar cursos en línea; esto es en modalidad a distancia, presencial o ambas.

Mcanally-Salas y Pérez-Fragoso (2000) proponen que las características para un curso en línea de apoyo tienen que contar con lo siguiente:

- Transmisión y acceso.

- Control.

- Interacción.

- Características simbólicas del medio.

- La presencia social creada a través del medio.

- La interface entre el usuario y la máquina.

La necesidad actual de aprendizaje ha llevado a usar diferentes formas y modelos de aprendizaje, dice al respecto el artículo uno de la declaración mundial sobre educación para todos.

En un extremo se ubican los cursos totalmente virtuales que dependen para todo de Internet, y en otro los cursos totalmente presenciales, que dependen para todo de la asistencia a clases; sin embargo, tanto la presencia puede ser enriquecida con los recursos de Internet, como la virtualidad puede ser fortalecida con encuentros personales.

Los cursos presenciales se ofrecen de manera convencional, se requiere la asistencia del profesor y del estudiante de acuerdo con el horario establecido al principio de cada semestre. El profesor desarrolla el curso y se apoya en los recursos que considera pertinentes, su característica principal es que todas las asistencias a clases son obligatorias.
Los cursos enriquecidos, son cursos presenciales que se enriquecen o se fortalecen con algunos recursos principales provenientes de Internet. Los recursos educativos en línea (usando Internet) pueden ser programas de estudio, lecturas, artículos, acervos virtuales, glosarios, etc. Son seleccionados o elaborados por el profesor para apoyar su curso presencial. Es posible que en esta modalidad sea conveniente (a criterio del profesor) sustituir alguna sesión presencial por actividades de consulta de recursos en línea, sin que sea una política de aplicación generalizada de la que se pueda llegar a buscar sin el sustento pedagógico necesario.

Los cursos mixtos son una combinación de actividades presenciales con actividades en línea, en donde, según Reigeluth (1999), el profesor tiene que ser más "un guía a nuestro lado" que "un sabio en el estrado". La cantidad de presencia a sesiones dependerá de la planeación y propósitos del curso. Así se puede pensar, por ejemplo, en que la teoría se puede revisar en línea y las prácticas realizarse en sesión presencial. Otra posibilidad es que se desarrollen, en lugar de cuatro horas de sesión, una sola de dos horas a cambio de algunas actividades realizadas vía Internet.

El plan de curso puede incluir también poner a disposición de los estudiantes toda la información como: objetivos, lecturas, guía de actividades, etc. Y poner una primera sesión de introducción al curso, establecer criterios de evaluación, y acordar otros detalles conjuntamente entre profesor y estudiante, otras sesiones se pueden programar con cierta periodicidad, sin caer en la modalidad de curso presencial.

\section{PLANTEAMIENTO DEL PROBLEMA}

Los principales retos implican identificar los factores necesarios para lograr $100 \%$ en la relación entre asignatura, acceso a los medio tecnológicos y acceso al MIEL, en un modelo presencial centrado en el aprendizaje, a la par que se rescatan los elementos que propician la diversidad de espacios en línea en este proceso, las implicaciones de la misma en entornos virtuales en una institución pública y sin dejar a un lado el encuentro del común denominador para los espacios en línea con material Instruccional, así como aquellos factores que afectan o influyen directa o indirectamente el nivel de los espacios en línea con material Instruccional como apoyo en el proceso de aprendizaje.

La Universidad de Guadalajara, a partir de la reforma de 1995, de ser una universidad 
centralizada en la zona metropolitana de Guadalajara, pasa a ser una universidad descentralizada y ubicada en las distintas regiones del estado de Jalisco, con un modelo departamental y matricial, en donde un departamento ofrece asignaturas a distintos programas educativos en cada uno de los centros regionales.

El CU Valles se encuentra ubicado en la región Valles del estado de Jalisco México, una región con escasas vías de comunicación, que en su mayoría se encuentran en condiciones precarias; en la que el $80 \%$ de la población tiene escasos recursos y presenta una gran demanda de capacitación. En este contexto, la Universidad de Guadalajara abrió el proyecto de descentralización en el estado de Jalisco, creando los centros regionales como el CU Valles en Ameca Jalisco, el Centro Universitario del Sur (en adelante CU Sur) ubicado en Ciudad Guzmán, el Sistema de Universidad Virtual (en adelante SUV) y cuatro centros regionales más, ubicados en los altos, lagos, y costa, buscando generar condiciones de equidad y atendiendo a la máxima población posible con los recursos disponibles.

En atención a la dispersión de la población estudiantil, los costos de traslado y las condiciones económicas, en CU Valles el modelo pedagógico se reestructuró. Uno de los elementos importantes en esta restructuración es el tiempo que el estudiante invierte en su proceso de aprendizaje en el aula, reduciendo al $50 \%$ este tiempo y el otro $50 \%$ distribuyéndolo en actividades extra-áulicas con el soporte de MIEL, a través de plataformas de gestión de la información y del aprendizaje, ofreciendo a los estudiantes en sus respectivas localidades CASAS Universitarias para reducir la brecha digital.

\section{DESARROLLO}

El modelo académico del CU Valles requiere el apoyo de materiales autoinstruccionales, con esto nos referimos a todos aquellos recursos instruccionales y didácticos que le permitan al estudiante de forma particular y en lo personal avanzar en su proceso de aprendizaje, y en ese sentido los recursos electrónicos adquirieron gran importancia. En 2004, la cantidad de recursos electrónicos se incrementó en $96 \%$ con relación a 2003, sin embargo entre el 2010 y el 2011 se perdió un $59.50 \%$ de los cursos existentes al 2010 (610) y actualmente se ofrecen 508, que equivalen al 83\% del máximo que se alcanzó en el 2010 (ver gráfico 1). En función de los resultados arrojados por los diagnósticos realizados semestralmente a todos los espacios con MIEL, existe una gran diversidad de estructuras y organización en los recursos electrónicos disponibles por asignatura, desde aquellos que únicamente los utilizan para establecer comunicación, hasta los que bosquejan un diseño Instruccional debidamente organizado y estructurado con material didáctico.

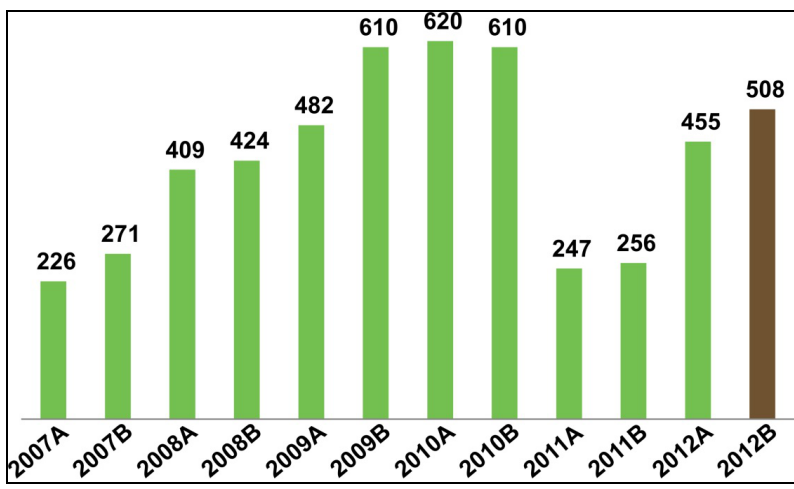

Gráfico 1. Cursos con MIEL

Fuente: Elaboración propia

Se requiere una estrategia para favorecer el tránsito de la comunidad estudiantil y académica a la sociedad del conocimiento, mediante el uso de las tecnologías de la información y comunicación (TIC), en la modernización y mejora de la calidad del sistema educativo a través del diseño Instruccional y material didáctico en línea y la posibilidad de tener acceso a los recursos tecnológicos necesarios para disponer de la información de la sociedad del conocimiento. Se tienen que analizar los procesos involucrados, los parámetros, comparar los recursos electrónicos, las estructuras de organización, los elementos evaluables, los esquemas de trabajo, y en función de la planeación y los contenidos temáticos de cada asignatura proponer un marco operativo que garantice la calidad y un buen seguimiento de las actividades por los participantes activos en cada curso, que guie a una evaluación objetiva del seguimiento académico, la atención y los logros obtenidos gracias a estos recursos.

Ante este panorama el CU Valles a través de su Coordinación de Tecnologías para el Aprendizaje (en adelante CTA) y la Coordinación de Extensión (en adelante CE) han establecido el programa de las CASAS Universitarias como una estrategia para facilitar el acceso a los recursos informáticos y de conexión a Internet a aquellos estudiantes y público en general que requieran de conectividad y que no cuenten con ello desde sus propios hogares. 
De acuerdo con las estadísticas proporcionadas por CTA, en Agosto de 2012 solamente el 56\% de los estudiantes del CU Valles cuentan con acceso a Internet, lo que propició el establecer mecanismos que permitieran facilitar el acceso a equipos tecnológicos para los estudiantes que así lo requirieran ayudando así a que su aprendizaje con apoyo de las plataformas de gestión de la información no se viera afectada (ver gráfico 2).

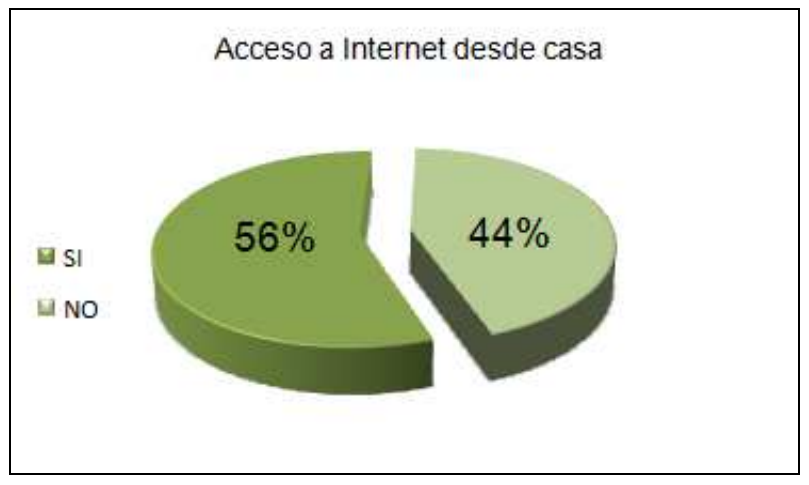

Gráfico 2. Porcentaje de Estudiantes de CU Valles con acceso a Internet desde sus hogares

Fuente: Elaboración propia

De esta manera surgen las CASAS Universitarias como una estrategia para reducir esa brecha entre quienes tienen y quienes no acceso a la red; Las CASAS son laboratorios de aprendizaje que se encuentran ubicados fuera del CU Valles y desde los cuales se pueden acceder a los recursos y materiales educativos que están hospedados en las plataformas informáticas del propio centro. Las CASAS son espacios que ofrecen a la comunidad servicios de información y comunicación.

Las Comunidades de Aprendizaje y Servicios Académicos funcionan en cooperación entre el Centro Universitario de los Valles y el Gobierno Municipal en el cual se encuentre ubicada cada CASA. El Gobierno Municipal facilita el espacio físico, local o domicilio así como una persona que funge como la responsable de la administración y aseo de la misma. El Centro Universitario proporciona el equipo tecnológico y el soporte técnico necesario para brindar servicios siempre funcionales. De esta manera es posible extender los servicios universitarios fuera de las instalaciones del centro, llevando hasta las comunidades las mismas funciones sustantivas de la Universidad como lo son la Investigación, la Docencia, la Extensión y la Vinculación mediante diferentes programas como lo son:

- Tutorías

- Asesorías Jurídicas.

- Asesorías Empresariales.
- Talleres y Diplomados de Educación Continua.

- Asesoría en cuestiones de Informática y Computación.

- Servicios Bibliotecarios.

- Materiales para el Aprendizaje de Idiomas.

- Asesoría en Servicios Turísticos (ver gráfico 3).

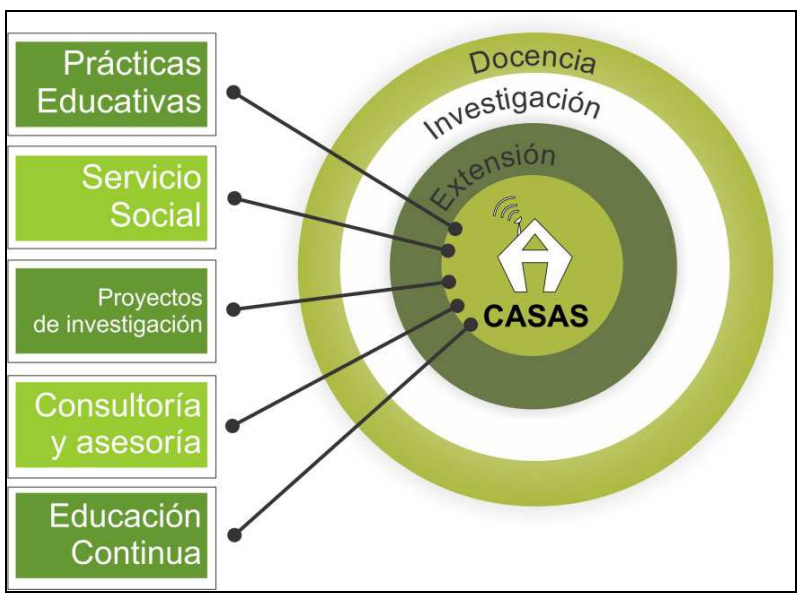

Gráfico 3. Funciones de Extensión de las CASAS hacia las comunidades de la Región Valles del Estado de Jalisco

Las CASAS Universitarias iniciaron sus actividades en 6 municipios del Estado de Jalisco, siendo éstos los que mayor matrícula registran y los más alejados geográficamente, municipios de incidencia pertenecientes a la región Valles de Jalisco. Estos municipios son: Ameca, Talpa de Allende, Tala, San Martín de Hidalgo, Ahualulco de Mercado así como Etzatlán Jalisco (ver gráfico 4).

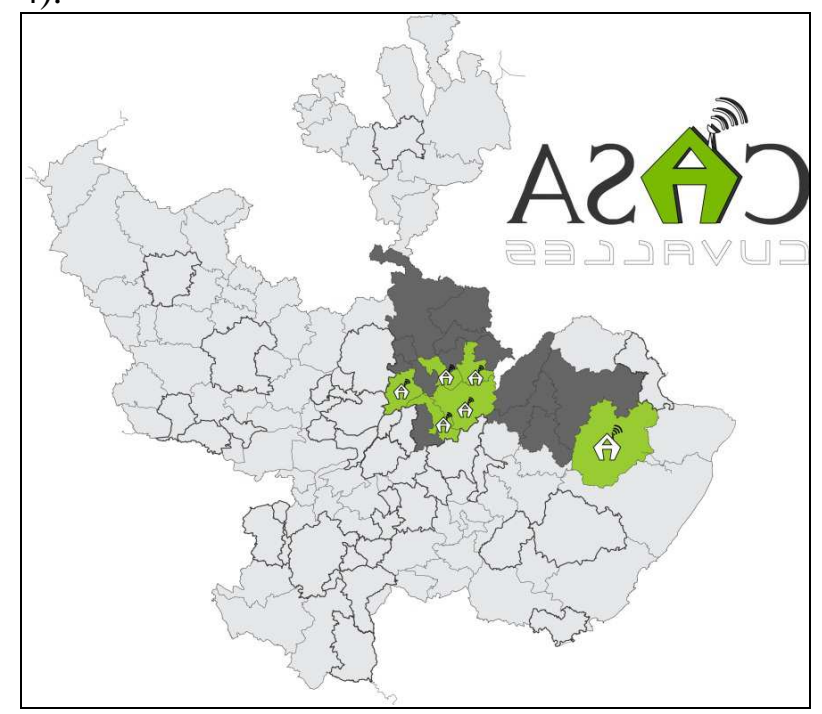

Gráfico 4. Comunidades de Aprendizaje y Servicios Académicos (CASA) en seis municipios de la Región Valles del Estado de Jalisco 
Cada CASA cuenta con 10 equipos de cómputo conectados a Internet, además tienen conexión inalámbrica para que si algún usuario opta por llevar su propio equipo portátil se puede conectar mediante WiFi. Además cuentan con equipos de fotocopiado, digitalizador de documentos y algunos de ellos video conferentes para establecer reuniones con audio y video con los académicos del Centro Universitario e incluso de otras Universidades.

Personal de la CTA es quien brinda soporte técnico a estos equipos de cómputo tanto en su hardware como en su software, se hacen visitas calendarizadas a los 6 municipios que cuenta con una CASA y se brinda servicio preventivo y correctivo a los equipos que lo requieran llevando una bitácora de lo realizado en cada una de ellas.

A mediano plazo, CU Valles buscará extender el programa CASA a los 19 municipios de influencia, la meta es que toda la Región de los Valles cuente con un espacio equipado con tecnología informática y con acceso a la red que permitan acercar a la mayoría de sus estudiantes al esquema de enseñanza - aprendizaje apoyado en las Tecnologías de la Información y Comunicación (ver gráfico 5)

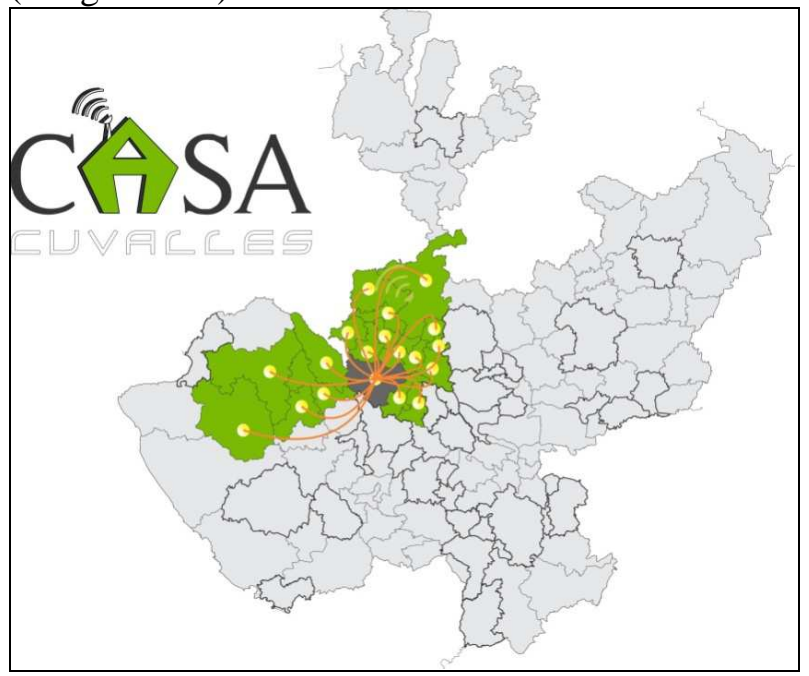

Gráfico 5. Municipios del Estado de Jalisco que contarán con una CASA a mediano plazo.

Actualmente el CU Valles cuenta con 4,313 estudiantes (ver gráfico 6), en su infraestructura dispone de 1,202 equipos de cómputo (ver gráfico $7)$, de los cuales el $68 \%$ está destinado al uso de los estudiantes (ver gráfico 8), cuenta con 6 CASAS Universitarias las cuales cuentan con 10 equipos de cómputo cada una.

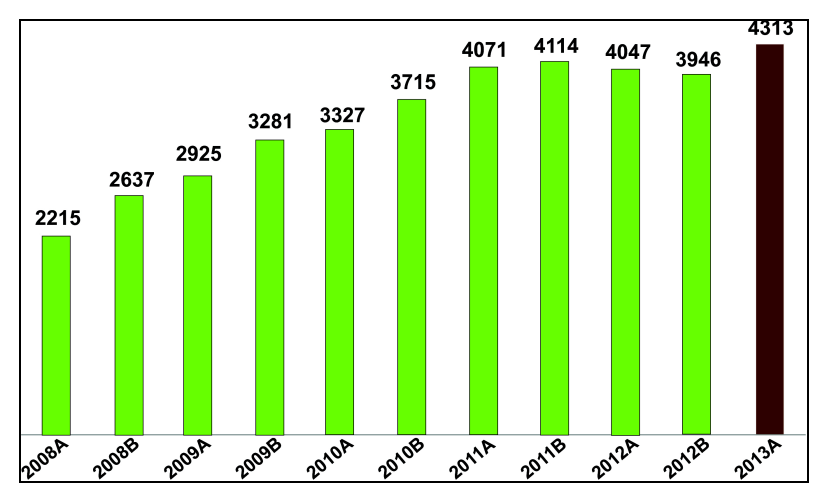

Gráfico 6. Estudiantes Matriculados por Ciclo Escolar en CU Valles

Fuente: Elaboración propia

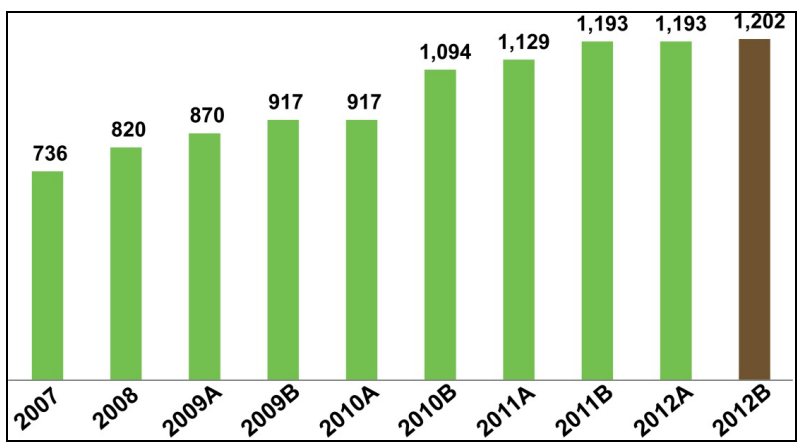

Gráfico 7. Equipo de Cómputo en CU Valles

Fuente: Elaboración propia

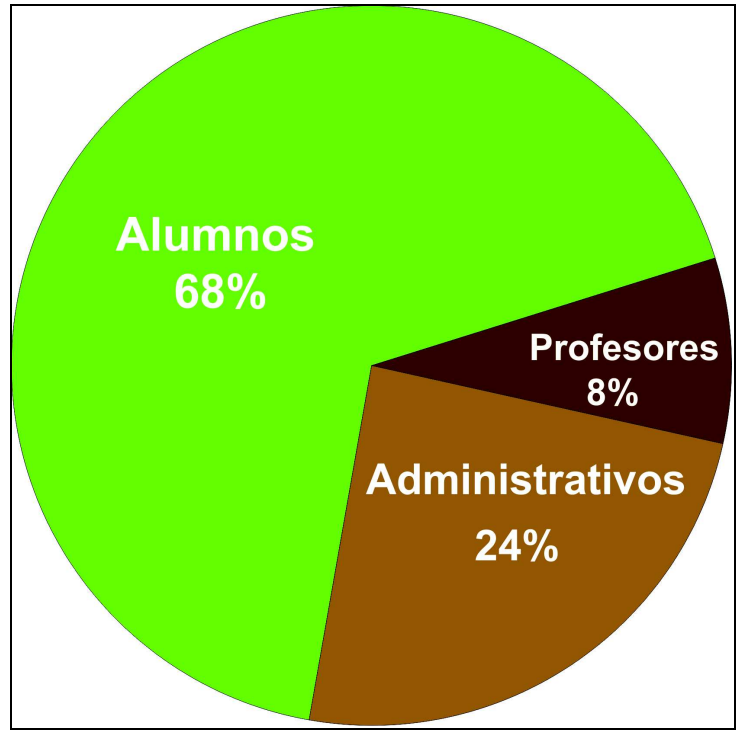

Gráfico 8. Distribución del Equipo de Cómputo en CU Valles

Fuente: Santana (2014)

De acuerdo a la disciplina en la que inciden los recursos electrónicos, existe una diferencia clara en el uso de los mismos.

Es fundamental el diseño Instruccional aprobado por las academias a través del trabajo colegiado, que garantice la mejora continua en la 
interacción estudiante-estudiante, estudianteprofesor, y el propio aprendizaje, ya que es el factor principal que muestra una medición eficiente en los cursos con MIEL, que no se vieron afectados sino, todo lo contrario: presentan una incidencia positiva en el modelo académico, aun cuando el cambio de instructor es continuo, semestre a semestre.

Actualmente, el CU Valles cuenta con una estrategia en apoyo a docentes en la construcción y actualización del MIEL como se muestra en el gráfico 9 y 10.

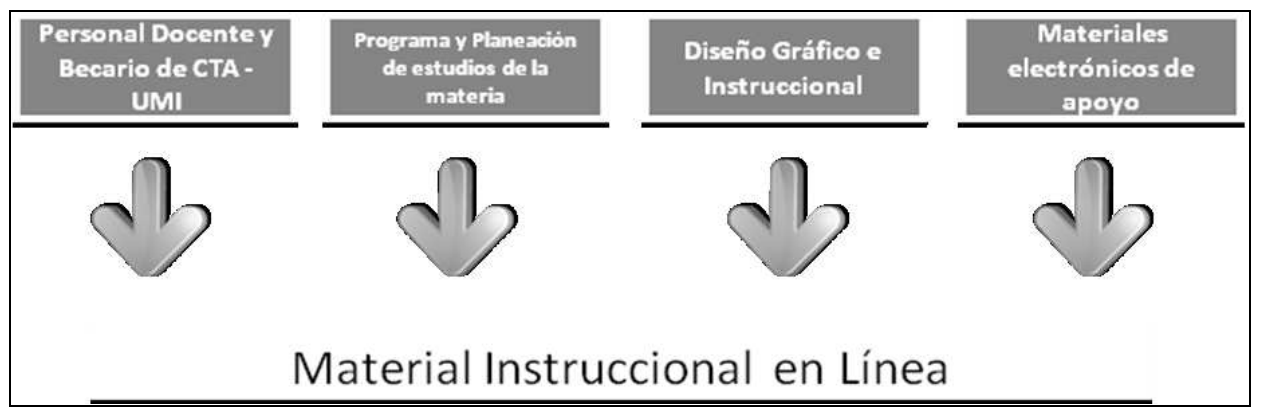

Gráfico 9. Diseño, construcción y mantenimiento de MIEL

Fuente: Elaboración propia

El modelo de operación institucional, contempla como base de interacción entre las unidades académicas responsables de los programas y contenidos de las asignaturas, académicas responsables de los programas y contenidos de las asignaturas, es indispensable la unidad de diseño, mantenimiento y construcción de espacios en línea con diseño Instruccional y material didáctico, un programa de capacitación continua en el área para garantizar el perfil adecuado, tanto de estudiantes, como de asesores, y además la unidad para la evaluación y seguimiento de las interacciones académicas a través de estos recursos, sin olvidar la necesidad de reconocer el esfuerzo personal e individual de los que dirigen los espacios con MIEL (ver gráfico $10)$.

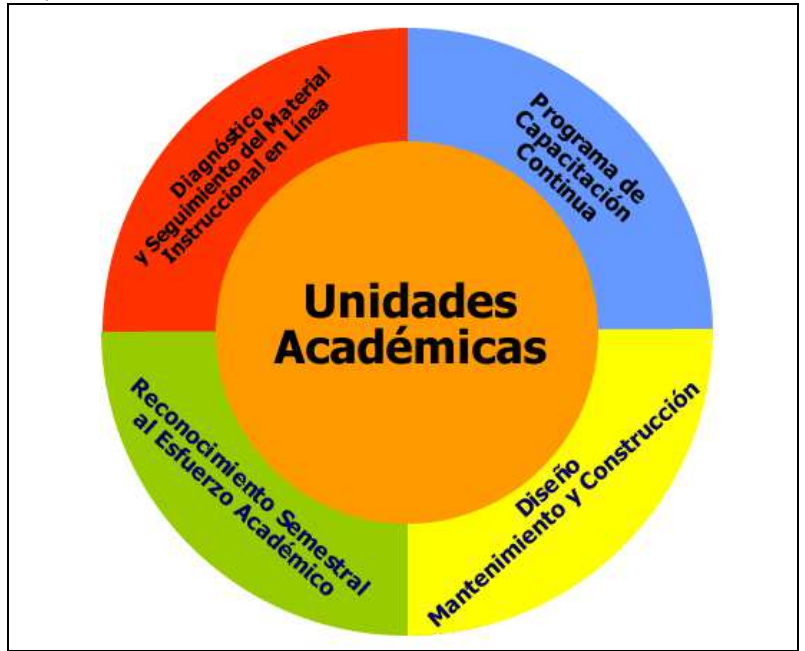

Gráfico 10. Modelo de operación institucional de CU Valles para el mantenimiento, construcción y evaluación de los recursos en línea, para optimizar la actividad presencial con MIEL.
Con la participación y opinión de académicos, estudiantes y administrativos, descartando las diferencias que pueden existir entre asignaturas tipo práctica o teórica, o las propias diferencias entre el área de las ciencias sociales y naturales, se encontró el común denominador para los espacios en línea con diseño Instruccional necesarios para los espacios en línea de un modelo presencial, enriquecido con tecnologías de información y comunicación, entre los cuales se destaca la importancia de la presentación del curso y asesor, programa, objetivos, criterios de evaluación, acreditación y calificación, planeación de actividades dentro y fuera del aula para las sesiones del ciclo escolar, herramientas de comunicación, buzones con instrucciones, calendario de actividades, lecturas, instrumentos de evaluación y prácticas o actividades de retroalimentación.

\section{CONCLUSIÓN}

En este trabajo se muestra que los efectos de los recursos electrónicos presentados y desarrollados en el CU Valles están íntimamente relacionados al desempeño académico en el modelo presencial optimizado centrado en el aprendizaje.

Los recursos electrónicos del CU Valles tratan de promover y asegurar la comunicación entre el asesor y el estudiante, para así salvar la dificultad de no estar presencialmente en todos los momentos del trabajo educativo que los relaciona. Para ello se ha recurrido al uso de diferentes tecnologías que faciliten la comunicación entre los estudiantes y el asesor, entrega de actividades, programación académica, exposición de los resultados. El énfasis 
en el uso actual de las tecnologías se enfoca en el apoyo o facilitación que se puede otorgar para lograr aprendizajes a través de las mismas.

En virtud de los resultados y siendo los recursos electrónicos una fortaleza del trabajo académico en el CU Valles, se implementó una estrategia que garantiza el desarrollo continuo de los recursos instruccionales en línea, utilizando un modelo de ambiente de aprendizaje dinámico que defienda, evalúe y monitoree la dependencia de las variables que intervienen en apoyo al modelo académico.

Actualmente el CU Valles cuenta con una gran diversidad de espacios en línea con recursos que se encuentran en función de las necesidades particulares en cada asignatura.

Si no existe un mecanismo claro de evaluación, si no se defiende en acuerdo y justificando lo que se espera, y cuáles son los elementos que se requieren, la diversidad surge en su conjunto como un esfuerzo por utilizar de forma óptima las tecnologías de información y comunicación para solventar necesidades particulares.

La diversidad de estrategias, y el uso diverso de los recursos es buena, pero lleva a contextos distintos si no se enmarcan de forma clara los beneficios que se esperan con el uso de los mismos; si buscamos calidad y resultados tangibles, es prudente sumar esfuerzos y no segmentarlos, definiendo el marco operativo, de evaluación y los beneficios, tanto para instructores, como para estudiantes que el mismo aporta.

Los factores que afectan el nivel del MIEL son: la formación y capacitación del personal docente y estudiantes en el uso y convicción de los aportes de las TIC, la cultura, falta de apoyo técnico especializado en diseño Instruccional $y$ en construcción de recursos en línea, los recursos tecnológicos disponibles; y los factores que influyen en la comunidad del uso y aprovechamiento de estos recursos por parte de los estudiantes se ven influenciados directamente por los principios didácticos como acogimiento, adecuación, flexibilidad metodológica, metocognición, motivación, interacción, proximidad, eficiencia, participación y construcción significativa del conocimiento.

\section{REFERENCIAS}

Alonso-Tapia, J. (1997). Orientación educativa. Madrid: Síntesis.

Ausubel, D, P. (1978). Educational Psychology, a Cognitive view. Nueva York: HoltyRinehart.
Bates, A, W. (2001). Cómo gestionar el cambio tecnológico. Estrategias para los responsables de centros universitarios. Barcelona: Ediouc. Gedisa.

Blanco-Méndez, N., y Carlos-Blanco, J. (2002). Desarrollo de un $\mathrm{CD}$ multimedia para el aprendizaje de los circuitos lógicos. Universidad de las palmas de Gran Canaria: V Congreso de Tecnologías a la enseñanza de la Electrónica (TAEE 2002).

Coll, C. (1992). Psicología y currículum. México: Paidós.

Fly-Jones, B., y Sederburge-Ogle, D. (1998). Estrategias para enseñar a aprender. Buenos Aires: Aique.

Giordan, A. (1998). Aprender. París: Débats Belin.

Hernández, H. P. (1991). Psicología de la educación. Corrientes actuales y teorías aplicadas. México: Trillas.

Majo, J., y Marques, P. (2002). La revolución educativa en la era en internet. Barcelona: Cisspraxis.

Mcanally-Salas, L. S., y Pérez-Fragoso, C. (2000). Diseño y evaluación de un curso en línea a nivel Licenciatura.

Monereo, C. (1993). Las estrategias de Aprendizaje. Doménech, Barcelona.

Pascual, M. P. (2003). El Blended learning reduce el ahorro de la formación on-line pero gana en calidad. Educaweb.com. Educación, formación y Trabajo, 69. Recuperado http://www.educaweb.com/esp/servicios/monografico/ formacionvirtual/1181108.asp

Pasturino, M. (1999). La construcción de competencias profesionales y laborales en los programas de inserción productiva. San Salvador: Cinterfor-OIT.

Prieto, M. D. (1993). El programa de enriquecimiento instrumental de Feuerstein. Madrid: Bruño.

Reigeluth, C. M. (1999). Diseño de la instrucción. Teorías y modelos. Madrid: Santillana.

Román-Pérez, M., y Díez-López, E. (2000). Aprendizaje y currículo. Diseños curriculares aplicados. Buenos Aires- México: Ediciones Novedades Educativas.

Santana-Medina, J. L. (2014). Informe de Actividades 2013 2014. Ameca: Edición interna del CU Valles.

Siordia, S. (2011). La Nueva Enseñanza, Tecnología y Sustentabilidad. En A. Ramón, R. Mora, L. Sulmont Haak, V. Sigal de los Santos, A. Vega, F. Maldonado, y otros, El papel de los dispositivos de normalización y los procesos de subjetivación en la educación moderna y sus repercusiones en la sustentabilidad (pp. 189-252). Zapopan, Jalisco, México: Umbral. b 